DOI: 10.12731/wsd-2017-4-205-218

УДК 616.37-006.6-092

\title{
ТАРГЕТНАЯ ТЕРАПИЯ У ПАЦИЕНТОВ С НЕЙРОФИБРОМАТОЗОМ (ОБЗОР ЛИТЕРАТУРЫ)
}

Жуковская Е.В., Бондаренко В.П., Спичак И.И., Сидоренко Л.В.

Актуальность представленного обзора обусловлена высокой частотой нейрофиброматоза 1 типа (NF1) и нейрофиброматоз 2 типа (NF2) в популяции и частой ассоциацией с развитием злокачественных новообразований. Целью подготовки обзора является анализ данных литературы, которые описывают результаты применения таргетных препаратов лечения новообразований у пациентов с нейрофиброматозом Результатами литературного поиска стало обобщение современных подходов к проведению таргетной терапии нейрофброматоза как NF1, так и NF2 типов. Представлены данные о фармакогенетике и принципах этиопатогенетической терапии с использованием следующих препаратов таргетной терапии: бевацизумаб, эрлотиниб, иматиниб, сорафениб., сиролимус. Сводная таблииа с результатами обзора содержит сведения из 39 литературных источников. По мнению большинства авторов, инновационная таргетная терапия нейрофиброматоза может оказаться эффективной у многих паџиентов с распространёнными формами заболевания.

Ключевые слова: нейрофиброматоз; NF1; NF2; таргетная терапия.

\section{TARGET THERAPY FOR PATIENTS WITH NEUROFIBROMATOSIS (LITERATURE REVIEW)}

\section{Zhukovskaya E.V., Bondarenko V.P., Spichak I.I., Sidorenko L.V.}

The relevance of the presented review is due to the high incidence of type 1 neurofibromatosis (NF1) and type 2 neurofibromatosis (NF2) in the population and frequent association with the development of malignant neoplasms. The purpose of the review is to analyze literature data that describe the results of the use of targeted drugs for the treatment of neoplasms in patients with 
neurofibromatosis. The results of the literary search became a generalization of modern approaches to targeted therapy of neurofromatosis both NF1 and NF2 types. The results of the literary search became a generalization of modern approaches to targeted therapy of neurofromatosis both NF1 and NF2 types. Data on pharmacogenetics and the principles of etiopathogenetic therapy with the use of the following targeted therapy are presented: bevacizumab, erlotinib, imatinib, sorafenib, sirolimus. The summary table with the results of the survey contains information from 39 literature sources. According to the majority of authors, innovative target therapy of neurofibromatosis can be effective in many patients with advanced forms of the disease.

Keywords: neurofibromatosis; NF1; NF2; target therapy.

\section{Введение}

Внутриклеточные механизмы развития заболеваний Нейрофиброматоза 1 типа (NF1) и нейрофиброматоз 2 типа (NF2) определяются мутациями в генах на 17 и 22 хромосомах соответственно. Продукт экспрессии этих генов - синтез белков нейрофибромина (ингибитор Ras-сигнального пути) и протеина Merlin (ингибитор сигнального пути Нippo), которые, влияя на транскрипцию, пролиферацию, дифференцировку, повышают выживаемость клеток. Образование аномальных белков приводит к формированию клинических симптомов NF, том числе и злокачественных новообразований [1, 2].

Учитывая системный характер NF проведение классической цитостатической и лучевой терапии по поводу проявлений пролиферативного синдрома в случае формирования неоперабельных опухолевых масс малоэффективно $[3,4,5]$.

Активные исследования механизмов опухолевой трансформации привели к идентификации сигнальных путей, ответственных за регуляцию пролиферации клеток. Инициируемые ростовыми факторами, гормонами, цитокинами сигнальные пути часто начинаются с тирозинкиназного каскада и заканчиваются активацией транскрипционных факторов, контролирующих экспрессию клеточного деления. Выявление таких активированных сигнальных путей и подавление их активности представляет одну из актуальных задач таргетной терапии NF.

\section{Препараты для таргетной терапии NF}

Препараты для нацеленной (таргетной) терапии - это вещества, которые воздействуют на конкретное звено в патогенетической цепи забо- 
левания - белки, рецепторы и другие вещества, имеющие критическое значение для развития новообразования. Для таргетной терапии, как правило, используются два типа молекул: моноклональные антитела и мелкие молекулы.

Препарат, который использовался в клинических испытаниях при лечении опухолей при NF - бевацизумаб. Бевацизумаб (bevacizumab) - торговое название Avastin - моноклональное антитело против фактора роста эндотелия сосудов (VEGF). VEGF - белок, фактор роста, который, связываясь со специфическим рецептором на клетке, индуцирует ангиогенез [6]. Опухолевая ткань, как известно, крайне чувствительна к васкуляризации, т.к., имея усиленный рост, она требует повышенного притока крови. Недостаток сосудов, а значит и поступления необходимых субстратов для роста ткани, существенно снижает прогрессию новообразований. Связываясь и образую белковый комплекс с VEGF бевацизумаб, не дает фактору роста эндотелия сосудов взаимодействовать с рецептором. Тем самым, бевацизумаб ингибирует рост сосудов [7].

В числе показаний к применению бевацизумаба различные онкологические заболевания: рак легкого, колоректальный рак, рак молочной железы, новообразования головного мозга.

Другой вид таргетной терапии - воздействие на клетку при помощи молекул меньшего размера - ингибиторов ферментов. Основными ингибиторами ферментов, имеющими клиническое значение, являются ингибиторы тирозин киназы. Тирозинкиназа - это фермент, подкласс протеинкиназ, осуществляющий перенос фосфатной группы с АТФ на белок. Фосфорилирование белков - один из важнейших механизмов передачи сигнала внутри клетки и регуляции клеточной активности, особенно клеточного деления. Различные тирозин киназы являются связующими звеньями в цепи превращений молекул внутри сигнальных путей. Ингибирование определенных тирозин киназ дает возможность регулировать сигнальные пути, поврежденные в раковых клетках [8]. Именно поэтому многие ингибиторы тирозин киназ взяты на вооружение в борьбе с онкологическими заболеваниями.

Эрлотиниб (Erlotinib) - торговое название Tarceva - препарат, относящийся к ингибиторам рецептора эпидермального фактора роста (EGFR). Предшественником эрлотиниба является гефитиниб (Iressa), который был первым препаратом этого типа. Механизм действия основан на специфическом ингибировании EGFR тирозинкиназы, которая усиленно экспрессируется и иногда повреждается вследствие мутации в опухолевых 
клетках. Эрлотиниб обратимым образом связывается с местом присоединения аденозин трифосфата на рецепторе [9]. Для передачи сигнала необходимо, чтобы две молекулы EGFR соединились с образованием гомодимера. В дальнейшем гомодимер фосфорилируется по тирозиновому остатку, что запускает каскад реакций фосфорилирования белков от клеточной стенки к ядру. Так происходит запуск многих клеточных процессов, в том числе роста и деления клеток. В клетках опухоли больного NF активация EGFR в числе прочих запускает каскад с дефектным белком, как при первом, так и при втором типе заболевания. При ингибировании тирозинкиназы образование фосфотирозинового остатка в EGFR не образуется, соответственно дальнейшая цепь биохимических реакций становится невозможной. Основным показанием к применению эрлотиниба является рак легкого. Этот препарат эффективен как при раке с наличием EGFR мутации, так и без нее, но наиболее положительный результаты наблюдается у больных с мутацией. В комбинации с гемцитабином эрлотиниб также используется при раке поджелудочной железы.

Следующий препарат группы ингибиторов киназ, участвовавший в клинических исследованиях среди больных NF - сорафениб. Сорафениб - торговое название Nexavar - это мультикиназный ингибитор нескольких киназ поверхности клеточной стенки (KIT, FLT-3, RET, VEGFR-1, VEGFR-2, VEGFR-3, and PDGFR-ß) и нескольких внутриклеточных киназ (Raf-киназы) [10]. Вышеупомянутые киназы участвуют в росте и делении опухолевой клетки и в ангиогенезе всей опухоли. Рецепторы факторов роста на поверхности клеточной стенки являются инициаторами, в первую очередь, роста сосудов, a Raf-киназы - это составляющая Ras-сигнального пути, ответственного за клеточный рост и деление.

Показаниями к применению сорафениба являются почечно-клеточный рак и печеночно-клеточный рак.

Иматиниб (imatinib) - торговое название Gleevec (Glivec) - ингибитор тирозинкиназы, препарат, используемый в терапии различных видов онкологических заболеваний. Основным назначением препарата является терапия положительных по Филадельфийской хромосоме $(\mathrm{Ph}+)$ вариантов хронического миелобластного и острого лимфобластного лейкозов [11]. При транслокации между 9 и 22 хромосомами возникает дефектная Филадельфийская хромосома, характерная для этих видов онкогематологических заболеваний. Результатом экспрессии одного из генов этой хромосомы является мутантный белок BCR-Abl. BCR-Abl является тирозинкиназой и, запуская ряд сигнальных путей, ингибирующих апоптоз, 
играет определяющую роль в канцерогенезе. BCR-Abl имеется только в опухолевых клетках, поэтому, ингибируя этот дефектный фермент, иматиниб таргетно воздействует на новообразование. Помимо ингибирования BCR-Abl иматиниб воздействует еще на ряд тирозинкиназ, в частности на c-Kit, что особенно важно для терапии NF. C-Kit (CD117) - тирозинкиназный рецептор к фактору стволовых клеток (SCF). В комплексе с лигандом с-Kit становится активным и запускает молекулярные сигнальные пути, направленные усиление выживаемости, деление и дифференцировку клеток. Мутация гена, кодирующего этот фермент наблюдается в ряде онкологических заболеваний. Роль с-Кit в патогенезе развития стромальных опухолей желудочно-кишечного тракта и нейрофибром при NF1 ясна не до конца, т.к. мутации гена не выявлены. Известно лишь, что активация c-Kit не связана с повышенной активностью Ras-сигнального пути $[12,13,14]$.

Салиразиб (Salirasib) - это экспериментальный препарат, дериват салициловой кислоты, потенциально обладающий антинеопластической активностью. Салиразиб перемещает любые изоформы Ras от их места прикрепления на клеточной мембране, тем самым предупреждает активацию Ras-сигнального пути, регулирующего клеточную пролиферацию, дифференцировку и старение $[15,16]$. Как известно, патология Ras- сигнального пути имеет важнейшее значение в патогенезе NF и многих онкологических заболеваний.

Лапатиниб (Lapatinib) - торговое название Tykerb - ингибитор тирозинкиназы HER2 (Human EGFR type 2) и EGFR. Ингибирование этих рецепторов ведет к прерыванию сигнала от них, молекулярные каскады, значимые в онкогенезе, прерываются. Лапатиниб блокирует HER2/EGFR путем связывания места прикрепления АТФ на этих протеин киназах, предотвращая дальнейшее фосфорилирование и запуск каскадного механизма [17].

Сиролимус природный антибиотик (рапамицин) впервые был выделен в 1975 г. С. Vezina и соавт. из Streptomyceshydroscopicus - торговое название Рапамун (Rapaminae) связывается со специфическим цитоплазматическим белком (FK-binding protein-12), комплекс FKPB-12-сиролимус подавляет активацию киназы «мишени рапамицина млекопитающих» (mTOR - mammalian target of rapamycin). Ингибирование mTOR приводит к блокаде нескольких специфических путей преобразования сигнала, и в конечном итоге к подавлению активации лимфоцитов и снижению иммунитета. Снижает активность Т- и В-лимфоцитов и подавляет оттор- 
жение аллогенного трансплантата. $\mathrm{mTOR}$, действует как главный выключатель многочисленных клеточных процессов, в том числе клеточного катаболизма и анаболизма, клеточной подвижности, ангиогенеза, и роста клеток. Сиролимус подавляет ответ на интерлейкин-2 и, таким образом, препятствует активации Т и В клеток, используется препарат в качестве иммуносупрессора [13]. Преклинические испытания на экспериментальных моделях и клиническое применение препарата демонстрируют перспективность препарата в отношении доброкачественных и злокачественных новообразований $[18,19]$.

\section{Клинические исследования таргетной терапии у больных с NF}

Для установления практической эффективности лечения был проведен анализ литературы клинических исследований препаратов для лечения опухолей у больных NF. Поиск литературы был проведен с помощью электронных ресурсов таких как PubMed, Clinical Key, Medscape и др. Результатом поиска стало описание клинических испытаний препаратов таргетного действия, указанных выше, у больных NF с новообразованиями различной локализации.

Из анализированных статей 7 являются клиническими испытаниями (Clinical Trials) - 7 полнотекстовых; 5 - описаний клинических случаев (Case Report) - 3 полнотекстовых источника и 4 выдержки; 2 - ретроспективные анализы (Retrospective Analysis) - 1 полнотекстовый источник и 1 выдержка из статьи; 1 выдержка из ретроспективного описания клинических случаев (Retrospective case review).

Иматиниб применялся в двух клинических испытаниях у больных NF1 с нейрофибромами и злокачественной опухолью оболочек периферических нервов (MPNST). Robertson et al. (2012) провели клиническое исследование с участием 36 пациентов с гроздевидными нейрофибромами. Дозировка препарата составляла 220 мг/м² дважды в день для детей и 400 мг дважды в день для взрослых. Результатом исследования стало уменьшение объема одной или нескольких нейрофибром у 12 из 36 пациентов (34\%) в течение 6 месяцев по данным МРТ. Самыми распространенными побочными эффектами оказались: нейтропения у 2-х пациентов, гипергликемия у 1-го пациента, повышение аминотрансферазы - у 1пациента [20]. Bano et al. после 4x месяцев терапии иматинибом отмечает стабилизацию объем опухоли (гроздевидная нейрофиброма, MPNST), уменьшение ее плотности, устранение гормон продуцирующей способности опухоли [21]. 
Применение Бевацизумаба описано в 7 статьях у больных NF2 с одной или несколькими вестибулярными шванномами. Plotkin et al. (2012) в исследовании у 31 пациента с NF2 и вестибулярными шванномами в ретроспективном анализе указывает, что в $57 \%$ случаев отмечалось снижение объема опухоли и в $55 \%$ замечено улучшение слуха при средней продолжительности лечения 14 месяцев [22]. В другом исследовании Plotkin et al. (2009) у 10 пациентов с тем же диагнозом демонстрирует снижение опухолевого объема у 9 пациентов, 6 имели улучшение при визуализации опухоли, у 4 из них оно поддерживалось на протяжении от 11 до 16 месяцев наблюдения. Медианой максимального снижения было $26 \%$ редукции опухоли. Семеро имели улучшение слуха. Зафиксировано всего 21 побочный эффект 1 и 2-ой степени тяжести [23].

Nunes et al. (2013) проводили клиническое исследование эффективности бевацизумаба у больных NF2 с менингиомами. Значимое уменьшение объема было достигнуто в 14 случаях среди 48 опухолей у 15 пациентов, по данным лучевой диагностики. Ответ на терапию не был длителен - в среднем 3,7 месяца, а время до прогрессии опухоли в среднем 15 месяцев [24].

Subbiah et al. (2012) в своем исследовании использовали различные препараты как по отдельности, так и в комбинациях у пациентов с NF2 и опухолями различной гистологической принадлежности. В этом исследовании бевацизумаб применялся у 4-х пациентов. У пациента с билатеральной акустической невромой, астроцитомой, эпиндимомой и менингиомой мозжечка бевацизумаб применялся после ряда препаратов, включавших AZD8330, сорафениб, эрлотиниб. После бевацизумаба был достигнут эффект стабилизации заболевания на срок более 9 месяцев. У пациента с множественными новообразованиями бевацизумаб применялся изначально как монотерапия, эффектом которой стала стабилизация заболевания более чем на 10 месяцев. Пациент с множественными шванномами и множественными опухолями периферических нервов принимал бевацизумаб в комплексе с темсиролимусом. Итогом лечения стала стабилизация заболевания более 4 месяцев. У пациента с билатеральной акустической невромой, шванномами T11, T12 и L1, множественными менингиомами головного мозга, эпиндимомой была применена та же терапия, в результате чего было достигнуто улучшение симптоматики, уменьшение объема опухоли на $33 \%$, и стабилизация заболевания более 9 месяцев. Типичными побочными эффектами для комплекса бевацизумаб + темсиролимус были протеинурия и гиперлипидемия. При монотерапия бевацизумабом побочных эффектов не отмечено [25]. 
Mautner et al. (2009) при описывает два клинических случая больных NF2, принимавших в качестве лечения бевацизумаб. По окончании курса отмечалось уменьшение опухоли более чем на $40 \%$ и существенное улучшение слуха после 6 месяцев терапии [26].

Eminowicz et al. (2011) приводят 2 клинических случая регрессии вестибулярных шванном после терапии бевацизумабом у пациентов NF 2, в анамнезе которых имеется лечение с помощью гамма-ножа [27]. Goutagny et al. приводит клинический случай лечения бевацизумабом больного с вестибулярной шванномой и париетальной менингиомой. Результатом лечения стало уменьшение в объеме шванномы на $15 \%$, а менингиомы на $22 \%$ после 15 недель лечения [28].

Эрлотиниб применялся авторами 3-х публикаций. Plotkin et al. (2010) проводил исследование среди 11 больных NF2 с вестибулярными шванномами. Из 10 доступных пациентов 3 показали максимальное уменьшение опухоли на $4 \%, 13 \%$ и $14 \% .8$ пациентов проходили обследование слуха режиме динамического наблюдения: 3 пациента отметили улучшение слуха, 3 - стабилизацию слуха, 2 - прогрессирующее снижение слуха. Побочное действие препарата проявлялось в виде сыпи, диареи, ломкости волос. Plotkin et al. (2008) также описывает клинический случай улучшения слуха от полной глухоты до звукоощущения (распознавание речи при помощи слухового аппарата) и уменьшение объема опухоли на $12 \%$ у пациента с вестибулярной шванномой после 11 месячного курса эрлотиниба.

Subbiah et al. (2012) использовал также эрлотиниб. У пациента с билатеральной акустической невромой, астроцитомой, эпиндимомой и менингиомой мозжечка применение эрлотиниба вместе с вальпроевой кислотой вызвало сонливость, из-за чего пришлось перейти на монотерапию эрлотинибом, которую пришлось прекратить из-за появления высыпаний после $2 \mathrm{x}$ месяцев курса. Терапия этого больного сорафенибом с вальпроевой кислотой также прекратилась в результате появления сыпи [25].

Сорафениб в клинических исследованиях применялся как для терапии NF1, так и для NF 2. Kim et al.(2012) использовал сорафениб для лечения 9 больных с NFII и гроздевидными нейрофибромами, уменьшения объема образований не последовало. Терапия сопровождалась большой степенью токсичности [29].

Лапатиниб применялся в исследовании 21-го больного NF 2 с вестибулярными шванномами. Karajannis et al. (2012) опубликовали результаты, указывающие на положительный ответ опухоли на терапию (17-из 21-го 
имели снижение объема в среднем на $23,5 \%$ ) и объективное улучшение слуха (13-больных в среднем на 30, 8\%) [30].

Салиразиб применялся только в одном клиническом исследовании у 2-х больных 2 в вышеупомянутом исследовании Subbiah et al. (2012). B обоих случаях наблюдалась стабилизация заболевания (4,5 и 10 месяцев) [25].

Сиролимус апробирован на широком перечне новообразований, это и типичные для NF нейрональные опухоли: шванномы, эпиндимомы, менгиомы и т.п., опухоли, обладающие секреторными функциями [31, 32, 33]. В представленных источниках пациенты как правило лишены шансов на радикальное хирургическое вмешательство из-за распространенности процесса и его локализации.

Возрастные критерии не являлись решающим признаком в выборе терапии, но чаще это лица от 3лет до 40 лет. Scott JR et al. (2013) продемонстрировал успешный контроль токсичности и передозировки сиролимуса у детей путем организации лекарственного мониторинга концентрации препарата. В эксперименте показана эффективность сиролимуса для коррекции костных поражений при NF 1 [34].

Учитывая сходные патогенетические механизмы формирования патологических симптомов при всех вариантах факоматозов исследователями, в том числе Oberg K. (2013) апробировали сиролимус и при туберозном склерозе. Также, как и у пациентов с NF эффективность препаратов sirolimus, everolimus, temsirolimus, and deforolimus оценивалась в качестве моно и комбинированной терапии у пациентов с туберозным склерозом [35].

\section{Заключение}

Все эти препараты теоретически должны воздействовать на патогенез и ингибировать рост опухоли у больных NF $[36,37,38]$. Но нельзя достоверно судить об эффективности этой терапии без проведения клинических испытаний. Анализируя результаты клинических испытаний NF препаратами для таргетной терапии, следует признать, что того количества клинических испытаний и исследований, которое существуют на сегодняшний день, недостаточно для проведения статистической обработки материала и получения четкого заключения об эффективности таргетной терапии в целом, на примере этих препаратов. Это невозможно из-за малого количества и неоднородности полученного материала.

Однако, большинство авторов положительно оценивают результаты терапии бевацизумаба. Во всех исследованиях с разной степенью эффективности был показан положительный результат терапии бевацизумабом 
у больных NF 2 с вестибулярными шванномами. Критериями успеха лечения были уменьшение объема опухоли и улучшение слуха. Также можно считать положительными результаты использования лапатиниба, эрлотиниба, сиролимуса и отчасти иматиниба, салазиба. Указание на имеющуюся токсичность софатениба, малочисленность исследуемой группы не позволяют пока отнести его к перспективным препаратам.

\section{Список литературы / References}

1. Blum R., Kloog Y. Tailoring Ras-pathway-Inhibitor combinations for cancer therapy. Drug Resistance Updates 8. 2005, pp. 369-380.

2. Staser K.I., Yang F.C., Clapp D.W. Pathogenesis of plexiform neurofibroma: tumor-stromal/ hematopoietic interactions in tumor progression. Annu Rev Pathol. 2012.7, pp. 469-95. doi: 10.1146/annurev-pathol-011811-132441. Epub 2011 Nov 7.

3. Zhukovskaya E.V., Berlinson M.Y., Basharova E.V. Malignant schwannoma in child with neurofibramatosis. Abstract book "Soft tissue sarcoma in children and adolescents" 3-rd International Congress Stuttgart April 30 - May 3-rd, 1997. 140.

4. Gottfried O.N., Viskochil D.H., Couldwel W.T. Neurofibromatosis Type 1 and tumorigenesis: molecular mechanisms and therapeutic implications. JNS January 2010, Vol. 28, No. 1, P. E8.

5. Спичак И.И., Жуковская Е.В., Моисеева Л.В., Башарова Е.В. Глиома зрительных нервов и хиазмы у пациента с нейрофиброматозом II типа. Иероглиф, 2005. том 8, № 27, Челябинск, C. 1100-1101 (Spichak I. I., Zhukovskaya E.V., Moiseeva L.V., Basharova E.V. Glioma of the optic nerves and chiasm in a patient with neurofibromatosis type II. Hieroglyph, 2005. vol. 8, No. 27, pp. 1100-1101).

6. Eremina V., Jefferson J.A., Kowalewska J. et al. VEGF Inhibition and Renal Thrombotic Microangiopathy. N. Engl. J. Med.2008 358(11), pp. 1129-1136.

7. Sliesoriaitis S., Tawfik B. Bevacizumab-induced bowel perforation. The Journal of the American Osteopathic Association. 2011.111(7), pp. 437-444.

8. Raymond E., Faivre S., Armand J. Epidermal growth factor receptor tyrosine kinase as a target for anticancer therapy. Drugs. 2000. 60(1), pp. 15-23.

9. Wilhelm S.M., Adnane L., Newell P., Villanueva A., Llovet J.M., Lynch M. Preclinical overview of sorafenib, a multikinase inhibitor that targets both Raf and VEGF and PDGF receptor tyrosine kinase signaling. Mol. Cancer Ther. 2008 7(10), pp. 3129-3140.

10. Maertens O., Prenen H., Debiec-Rychter M. Molecular pathogenesis of multiple gastrointestinal stromal tumors in NF1 patients. Hum Mol Genet. 2006 15(6), pp. 1015-1023. 
11. Fausel C. Targeted chronic myeloid leukemia therapy: Seeking a cure. Am J Health Syst Pharm. 2007 64, pp. 9-15.

12. Trovy-Marqui A.B., Tajara E.H. Neurofibromin: a general outlook. Cliical Genetics; 2000 70(1), pp. 1-13.

13. NF2 neurofibromin 2 (merlin) [Homo sapiens (human) ]. National Center for Biotechnology Information. URL: http://www.ncbi.nlm.nih.gov/gene/4771

14. Sainio M., Zhao F., Heiska L. Turunen O. et al. Neurofibroamatosis 2 tumor suppressor protein localizes with ezrin and CD44 and associates with actin-containing cytoskeleton. J. Cell Biol. 1997: 2249-2260.

15. Salirasib. NCI Drug Dictionary. National Cancer Institute. URL: http://www. cancer.gov/drugdictionary?cdrid=573226.

16. Riely G.J., Johnson M.L., Medina C., Rizvi N.A., Miller V.A., Kris M.G.Pietanza M.C., Azzoli C.G., Krug L.M., Pao W., Ginsberg M.S. A phase II trial of Salirasib in patients with lung adenocarcinomas with KRAS mutations. J Thorac Oncol. 2011;6(8), pp. 1435-1437.

17. Nelson M.H., Dolder C.R. Lapatinib: a novel dual tyrosine kinase inhibitor with activity in solid tumors. Ann Pharmacother. 2006; 40(2): 261-269.

18. Pachow D., Andrae N., Kliese N. et al. mTORC1 inhibitors suppress meningioma growth in mouse models. Clin Cancer Res. 2013 Mar 1; 19(5), pp. 1180-1189.

19. Banerjee S., Gianino S.M., GAO F. Et Al. Interpreting mammalian target of rapamycin and cell growth inhibition in a genetically engineered mouse model of Nf1-deficient astrocytes. Mol Cancer Ther. 2011 Feb; 10(2), pp. 279-291.

20. Robertson K.A., Nalepa G., Yang F.C. et al. Imatinib mesylate for plexiform neurofibromas in patients with neurofibromatosis type 1: a phase 2 trial. The Lancet Oncology, Volume 13, Issue 12, pp. 1218-1224, December 2012 PMID: 23099009.

21. Bano G., Mir F., Schey S. et al. A Novel Medical Treatment of Cushing's Due to Ectopic ACTH in a Patient With Neurofibromatosis Type 1. Int J Endocrinol Metab. 2013 winter; 11(1), pp. 52-56. Published online Dec 21, 2012.

22. Plotkin S.R., Merker V.L., Halpin C., et al. Bevacizumab for progressive vestibular schwannoma in neurofibromatosis type 2: a retrospective review of 31 patients. Otol Neurotol. 2012 Aug; 33 (6), pp. 1046-1052.

23. Plotkin S.R., Stemmer-Rachamimov A.O., Barker F.G. et al. Hearing improvement after bevacizumab in patients with neurofibromatosis type 2. N Engl J Med. 2009 Jul 23; 361 (4), pp. 358-367.

24. Nunes F.P., Merker V.L., Jennings D. et al. Bevacizumab treatment for meningiomas in NF2: a retrospective analysis of 15 patients. PLoS One. 2013;8(3): e59941. Epub 2013 Mar 21. 
25. Subbiah V., Slopis J., Hong D.S. et al. Treatment of patients with advanced neurofibromatosis type 2 with novel molecularly targeted therapies: from bench to bedside. J Clin Oncol. 2012 Feb 10;30 (5):e64-8. doi: 10.1200/JCO.2011.38.2614. Epub 2011 Dec 27.

26. Mautner V.F., Nguyen R., Kutta H. et al. Bevacizumab induces regression of vestibular schwannomas in patients with neurofibromatosis type 2. Neuro Oncol. 2010 January; 12(1), pp. 14-18. Published online 2009 October 20. doi: 10.1093/neuonc/nop010.

27. Eminowicz G.K., Raman R., Conibear J., Plowman P.N. Bevacizumab treatment for vestibular schwannomas in neurofibromatosis type two: report of two cases, including responses after prior gamma knife and vascular endothelial growth factor inhibition therapy. J Laryngol Otol. 2012 Jan;126 (1), pp. 79-82. doi: 10.1017/S0022215111002805. Epub 2011 Oct.

28. Goutagny S., Raymond E., Sterkers O. et al. Radiographic regression of cranial meningioma in a NF2 patient treated by bevacizumab. Ann Oncol. 2011 Apr;22(4 ), pp. 990-991. doi: 10.1093/annonc/mdr012. Epub 2011 Feb 28.

29. Kim A., Dombi E., Tepas K., Fox E. et al. Phase I trial and pharmacokinetic study of sorafenib in children with neurofibromatosis type I and plexiform neurofibromas. Pediatr Blood Cancer. 2013 Mar;60(3), pp. 396-401. doi: 10.1002/ pbc.24281. Epub 2012 Sep 7.

30. Karajannis MA, Legault G, Hagiwara M et al. Phase II trial of lapatinib in adult and pediatric patients with neurofibromatosis type 2 and progressive vestibular schwannomas. Neuro Oncol. 2012 Sep;14(9), pp. 1163-1170. doi: 10.1093/ neuonc/nos146. Epub 2012 Jul 27.

31. Anjum R., Blenis J. The RSK family of kinases: emerging roles in cellular signaling. Nature Reviews Molecular Cell Biology. 2008; 9(10), pp. 747-758.

32. Liu N., Xu N., Wei L.H., Chai G.L. Mammalian target of rapamycin inhibitor abrogates abnormal osteoclastogenesis in neurofibromatosis type 1. .Chin Med J (Engl). 2013 Jan; 126(1), pp. 101-107.

33. Mendoza M.C., Er E.E., and Blenis J. The Ras-ERK and PI3K-mTOR pathways: cross-talk and compensation. Trends in bioch. sciences. 2011; 36(6), pp. 320-328.

34. Scott J.R., Courter J.D., Saldaña S.N., Widemann B.C., Fisher M., Weiss B., Perentesis J., Vinks A.A. Population pharmacokinetics of sirolimus in pediatric patients with neurofibromatosis type 1.Ther Drug Monit. 2013 Jun; 35(3), pp. 332-337.

35. Öberg K. The genetics of neuroendocrine tumors. Semin Oncol. 2013 Feb; 40(1): 37-44. doi: 10.1053/j.seminoncol.2012.11.005.

36. Krueger D.A., Care M.M., Holland K. et al. Everolimus for subependymal giant-cell astrocytomas in tuberous sclerosis. New Eng. J. Med 2010; 363, pp. 1801-1811. 
37. Bertini E., Oka T., Sudol M., Strano S., Blandino G. YAP: at the crossroad between transformation and tumor suppression. Cell Cycle. 2009; 8(1), pp. 49-57.

38. Raymond E., Faivre S., Armand J. Epidermal growth factor receptor tyrosine kinase as a target for anticancer therapy. Drugs. 2000; 60(1), pp. 15-23.

\section{ДАННЫЕ ОБ АВТОРАХ}

Жуковская Елена Вячеславовна, д.м.н., зав отдела изучения токсических эффектов противоопухолевой терапии Лечебно-реабилитационного центра «Русское поле»

НМИЦ ДГОИ им. Дмитрия Рогачева МЗ России

ул. Саморы Машела, 1, г. Москва, 117198, Российская Федеращия

E-mail:elena_zhukovskay@mail.ru

Бондаренко Владислав Павлович, м.н.с. отдела изучения токсических эффектов противоопухолевой терапии ЛРНЦ «Русское поле» НМИЦ ДГОИ им. Дмитрия Рогачева МЗ России ул. Саморы Машела, 1, г. Москва, 117198, Российская Федераџия vlad-bondr@yandex.ru

Спичак Ирина Ильинична, д.м.н., доцент, зав. областным детским центра онкологии и гематологии

Областная детская клиническая больница им. Валентина Герайна ул. Блюхера, 42a, г. Челябинск, 454076, Российская Федераџия irina_spichak@mail.ru

Сидоренко Людмила Васильевна, к.м.н., зав. отделом мониторирования клинических исследований

НМИЦ ДГОИ им. Дмитрия Рогачева МЗ России

ул. Саморы Машела, 1, г. Москва, 117198, Российская Федерачия monami1511@rambler.ru

\section{DATA ABOUT THE AUTHORS}

Zhukovskaya Elena Vaycheslavovna, MD, Professor, Head of the Department of the Study of the Toxic Effects of Antitumor Therapy Scientific Rehabilitation Centre "Russian field"

National Medical Research Center of Pediatric Hematology, Oncology and Immunology named after Dmitry Rogachev, Health Ministry of Russia 1, Samor Mashel Str., Moscow, 117198, Russian Federation 
elena_zhukovskay@mail.ru

SPIN-code: 8225-6360

ORCID: 0000-0002-6899-7105

Bondarenko Vladislav Pavlovoch, Researcher in the Department of the Study of the Toxic Effects of Antitumor Therapy Scientific Rehabilitation Centre "Russian field"

National Medical Research Center of Pediatric Hematology, Oncology and Immunology named after Dmitry Rogachev, Health Ministry of Russia

1, Samor Mashel Str., Moscow, 117198, Russian Federation vlad-bondar@yandex.ru

SPIN-code: 8760-5180

Spichak Irina Ilinichna, MD, Assistant Professor, Head of Centre of Pediatric Hematology

Regional Children's Clinical Hospital named after Valentin Gerayn 42, Blucher Str., Chelaybinsk, 454076, Russian Federation irina_spichak@mail.ru

Sidorenko Ludmila Vasilevna, MD, Head of the Department Monitoring the Scientific Investigations

National Medical Research Center of Pediatric Hematology, Oncology and Immunology named after Dmitry Rogachev, Health Ministry of Russia

1, Samor Mashel Str., Moscow, 117198, Russian Federation monami1511@rambler.ru 